\title{
A Universal Binding Mechanism in Molecular Covalent Bonding and Nucleon-Nucleon Interaction
}

\author{
Nicolae Bogdan Mandache \\ National Institute for Laser, Plasma and Radiation Physics, Magurele, Romania \\ Email: mandache@infim.ro
}

How to cite this paper: Mandache, N.B. (2021) A Universal Binding Mechanism in Molecular Covalent Bonding and Nucleon-Nucleon Interaction. Journal of Modern Physics, 12, 1237-1247. https://doi.org/10.4236/jmp.2021.129076

Received: June 18, 2021

Accepted: July 9, 2021

Published: July 12, 2021

Copyright $\odot 2021$ by author(s) and Scientific Research Publishing Inc. This work is licensed under the Creative Commons Attribution International License (CC BY 4.0).

http://creativecommons.org/licenses/by/4.0/

\section{(c) (i) Open Access}

\begin{abstract}
In the hydrogen molecular ion, the kinetic energy lowering of the electron is associated with its delocalization due to electron exchange between the two protons of the molecule. This decrease in the kinetic energy of the exchanged electron in the hydrogen molecular ion and the decrease in the dynamical mass of the two exchanged pions in the nucleon-nucleon interaction are at the origin of the attraction mechanism in the molecular covalent bonding and in the nuclear interaction. Based on this unitary approach of the attraction mechanism, the formulas of molecular potential and central nucleon-nucleon potential were derived. The decrease in the mass of the exchanged pions in the nucleon-nucleon bound state, actually means the decrease in the mass of the nucleons. This nucleon mass decrease could be a manifestation of the partial chiral symmetry restoration in nuclear matter.
\end{abstract}

\section{Keywords}

Electron and Pion Exchange, Dynamical Mass, Molecular and Central Nucleon-Nucleon Potentials, Nucleon Mass Decrease, Partial Chiral Symmetry Restoration

\section{Introduction}

The mechanism of molecular covalent bonding by electron exchange and the mechanism of nucleon-nucleon attraction by pion exchange are important in our understanding of bound states.

In the hydrogen molecular ion $\mathrm{H}_{2}^{+}$, the kinetic energy lowering of the electron associated with its delocalization due to electron exchange between the two protons of the molecule, plays a fundamental role in the mechanism of covalent 
bonding [1] [2] [3]. The maximum molecular attraction is realized in the diatomic molecular bond by exchange of two electrons [1] [3]. This is the case of the hydrogen molecule $\mathrm{H}_{2}$ in which the two electrons are exchanged between the two protons.

Heisenberg was the first who presented the attractive force between proton and neutron in analogy to that in the hydrogen molecular ion $\mathrm{H}_{2}^{+}$[4]. Yukawa was the first who proposed the pion as the particle exchanged in the nucleon-nucleon interaction. The basic mechanism of nuclear attraction at intermediate range is still under debate. The two pion exchange seems to be the most favored candidate [4] [5] [6] [7] [8].

Feynman made a simple and unitary analysis of the mechanism of both covalent bonding and nucleon-nucleon interaction [3]. The probability amplitude for a particle to get from one place to another a distance $R$ is the key ingredient used in [3] to describe the behavior of the exchanged particle, the electron in the covalent bonding and the pion in the nucleon-nucleon interaction. The interaction energy is proportional to this probability amplitude [3].

Starting from Feynman analysis, a quantitative approach to the mechanism of nucleon-nucleon attraction was presented in [9] [10]. By analogy with the decrease in the kinetic energy of the exchanged electron in the molecule (a decrease of dynamical mass from a relativistic point of view), it has been proposed that the decrease in the dynamical mass of exchanged pions in the nucleon-nucleon interaction is the main mechanism responsible for nucleon-nucleon attraction. The delocalization of exchanged pions is strongly limited by the probability of pions to tunnel from one nucleon to another. A formula for the central nucleon-nucleon potential, which does not contain any parameter, was derived [10]. The probability concept was used instead of the probability amplitude.

In the present paper, using the concept of probability amplitude as in [3], both the potential of the hydrogen molecular ion $\mathrm{H}_{2}^{+}$(Section 2) and the central potential of the nucleon-nucleon interaction due to two pion exchange (Section 3) are derived. The two formulas of the potentials do not contain any unknown parameter. A universal binding mechanism in the molecular covalent bonding and in the central nucleon-nucleon interaction is demonstrated.

A possible manifestation of partial chiral symmetry restoration in nuclear matter is analyzed.

\section{Molecular Covalent Bonding}

In the hydrogen molecular ion $\mathrm{H}_{2}^{+}$, since there are two protons, there is more space where the electron can have a low potential energy than in the case of hydrogen atom. The exchanged electron spreads out lowering its kinetic energy, in accord with uncertainty relation. This kinetic energy decrease is at the origin of the molecular attraction in covalent bond, in particular in the $\mathrm{H}_{2}^{+}$ion [1] [2] [3]. 
A simple analysis of this binding mechanism of hydrogen molecular ion $\mathrm{H}_{2}^{+}$ is presented by Feynman in [3]. For large distances between the two protons of the $\mathrm{H}_{2}^{+}$ion the electrostatic potential energy of the exchanged electron is nearly zero over most of the space between the protons and the electron moves nearly like a free particle in empty space but with "negative" kinetic energy [3]:

$$
\frac{p^{2}}{2 m}=-W_{H} .
$$

where $W_{H}$ is the binding energy $(13.6 \mathrm{eV})$ of the hydrogen atom. This means that $\mathrm{p}$ is an imaginary number:

$$
p=i \sqrt{2 m_{e} W_{H}} .
$$

The probability amplitude $A$ for a particle of definite energy to get from one place to another a distance $R$ away is proportional to [3]:

$$
A \sim \frac{\mathrm{e}^{(i / \hbar) p R}}{R} .
$$

If the particle goes in one direction the probability amplitude is [3]:

$$
A \sim \mathrm{e}^{(i / \hbar) p R} .
$$

Replacing $\mathrm{p}$ (Formula (2)) one obtains that the amplitude of jumping of electron from one proton to the other proton of the molecule, will vary as [3]:

$$
A \sim \mathrm{e}^{-\frac{1}{\hbar}\left(\sqrt{2 m_{e} W_{H}}\right) R}=\mathrm{e}^{-R / a_{0}} .
$$

where $a_{0}=\hbar / \sqrt{2 m_{e} W_{H}}$ is the Bohr radius. In other words $A$ is the probability amplitude that the bound electron would penetrate the barrier in the space between the two protons. One can note that this exponential is just the wave function (excepting a normalization constant) of the fundamental level of the hydrogen atom. The interaction energy due to electron exchange is proportional to $A$ [3].

Starting from this approach presented in [3] let's make an estimation of the kinetic energy lowering of the exchange electron in the $\mathrm{H}_{2}^{+}$ion. When a hydrogen atom approaches a proton, they exchange the electron which tunnels back and forth between the two protons. This is equivalent with a slight delocalization of the electron from a region of characteristic dimension $a_{0}$ associated with $\mathrm{H}$ atom, to a region of characteristic dimension $a_{0}+\Delta(R)$ associated with the molecular ion $\mathrm{H}_{2}^{+}$, where $R$ is the distance between the two protons. This means that the mean kinetic energy of the exchanged electron in $\mathrm{H}_{2}^{+}$ion is lower than its mean kinetic energy in the $\mathrm{H}$ atom.

The slight delocalization $\Delta(R)$, is direct proportional to the distance $R$ between the two protons and is strongly limited by the probability amplitude (5) of the electron to penetrate the potential barrier between the two protons. From a physical point of view one expects that this probability amplitude of transmission is 1 for a barrier width $R \rightarrow 0$. Therefore, $\Delta(R)$, which is proportional both to the distance $R$ between the protons and to the probability amplitude of transmission of the exchanged electron, can be written as: 


$$
\Delta(R)=R \mathrm{e}^{-R / a_{0}} .
$$

The kinetic energy of the delocalized electron in $\mathrm{H}_{2}^{+}$ion is:

$$
E_{k i n, H_{2}^{+}}=\frac{p^{2}}{2 m_{e}}=\frac{\hbar^{2}}{2 m_{e}\left(a_{0}+\Delta(R)\right)^{2}}=\frac{\hbar^{2}}{2 m_{e}\left(a_{0}+R \mathrm{e}^{-R / a_{0}}\right)^{2}} .
$$

where $p=\hbar /\left[a_{0}+\Delta(R)\right]$ from uncertainty relation, and $\Delta(R)$ is given by relation (6).

The mean kinetic energy of electron in the $\mathrm{H}$ atom $E_{k i n, H}$ is:

$$
E_{k i n, H}=\frac{\hbar^{2}}{2 m_{e} a_{0}^{2}} .
$$

The decrease of the kinetic energy of the electron bound into the $\mathrm{H}_{2}^{+}$ion (7) compared to the kinetic energy of the electron bound into the $\mathrm{H}$ atom (8) is directly related to the formation of the molecular bound state [1] [2] [3]. The negative quantity $T(R)$ :

$$
\begin{aligned}
T(R) & =\frac{\hbar^{2}}{2 m_{e}\left(a_{0}+R \mathrm{e}^{-R / a_{0}}\right)^{2}}-\frac{\hbar^{2}}{2 m_{e} a_{0}^{2}} \\
& =-\frac{\hbar^{2}}{2 m_{e} a_{0}^{2}}\left(\frac{R}{a_{0}} \exp \left(-R / a_{0}\right)\right) \frac{2+\frac{R}{a_{0}} \exp \left(-R / a_{0}\right)}{\left(1+\frac{R}{a_{0}} \exp \left(-R / a_{0}\right)\right)^{2}}
\end{aligned}
$$

represents the main contribution to the attraction mechanism in the molecular $\mathrm{H}_{2}^{+}$ion.

The curve $T(R)$ is shown in Figure 1. The distance $R$ between the two protons is given in $a_{0}$ units. This curve is similar to those obtained by detailed quantum mechanics calculus [1] [2].

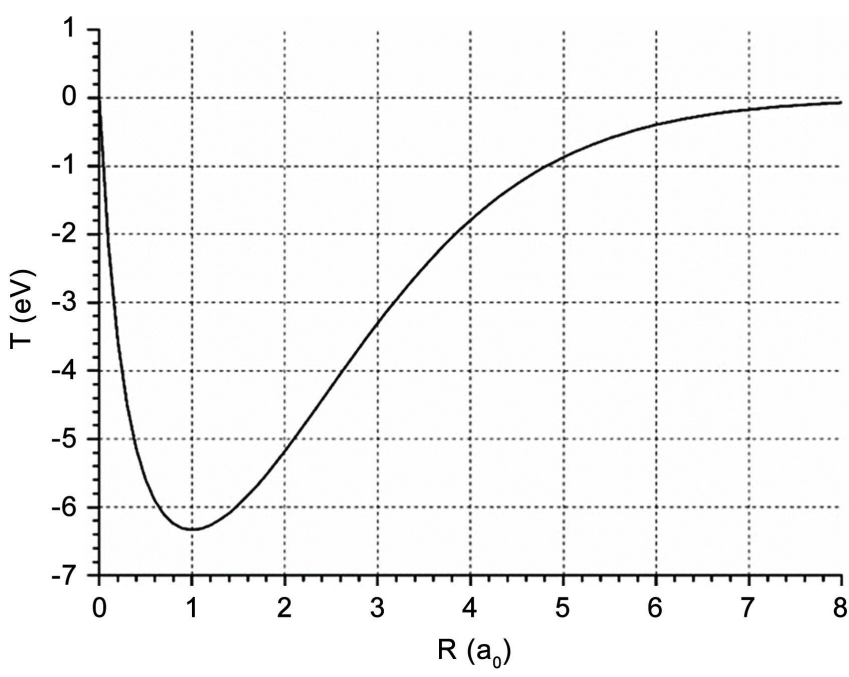

Figure 1. The decrease of the kinetic energy $T(R)$ of the exchanged electron in $\mathrm{H}_{2}^{+}$ion relative to its kinetic energy in the $\mathrm{H}$ atom. The distance $R$ between the two protons is in $a_{0}$ units. 
To obtain the total energy $E(R)$ of the $\mathrm{H}_{2}^{+}$ion one must add to $T(R)$ the contribution of the electrostatic interaction energy. The electron jumps from one proton to another, so it can be approximated that in the $\mathrm{H}_{2}^{+}$ion there is always a $\mathrm{H}$ atom (proton surrounded by the electron cloud) that interacts electrostatically with the other proton. Therefore the electrostatic repulsion between the two protons of the $\mathrm{H}_{2}^{+}$ion is partially reduced by the electron cloud. An estimation of this interaction can be obtained starting from the time-averaged potential of the (neutral) $\mathrm{H}$ atom [11]:

$$
\phi_{H}(R)=\frac{e}{a_{0}} \frac{\exp \left(-2 R / a_{0}\right)}{R / a_{0}}\left(1+\frac{R}{a_{0}}\right)
$$

The energy due to electrostatic interaction between the $\mathrm{H}$ atom and the other proton is $e \cdot \phi_{H}(R)$. The total energy of the $\mathrm{H}_{2}^{+}$ion is:

$$
E(R)=T(R)+e \cdot \phi_{H}(R) .
$$

and is shown in Figure 2. The distance $R$ between the two protons is given in $a_{0}$ units.

In Figure 2, the value of the minimum energy is $B=-4.4 \mathrm{eV}$ and the position of the minimum is at $R=2 a_{0}$. The experimental values are $B_{\text {exp }}=-2.8 \mathrm{eV}$ and $R_{\text {exp }}=1.06 \AA \cong 2 a_{0}$.

In a similar way one can analyses the covalent bond of $\mathrm{H}_{2}$ molecule in which two electrons are exchange. The probability amplitude (5) of exchange of an electron will be replaced by the probability amplitude of simultaneous exchange of the two electrons between the $\mathrm{H}$ atoms [3];

$$
A_{2 e} \sim \mathrm{e}^{-R / a_{0}} \cdot \mathrm{e}^{-R / a_{0}}=\mathrm{e}^{-2 R / \lambda_{\pi} a_{0}} .
$$

The lowering of the kinetic energies of both electrons gives the main contribution to the attraction mechanism in the $\mathrm{H}_{2}$ molecule.

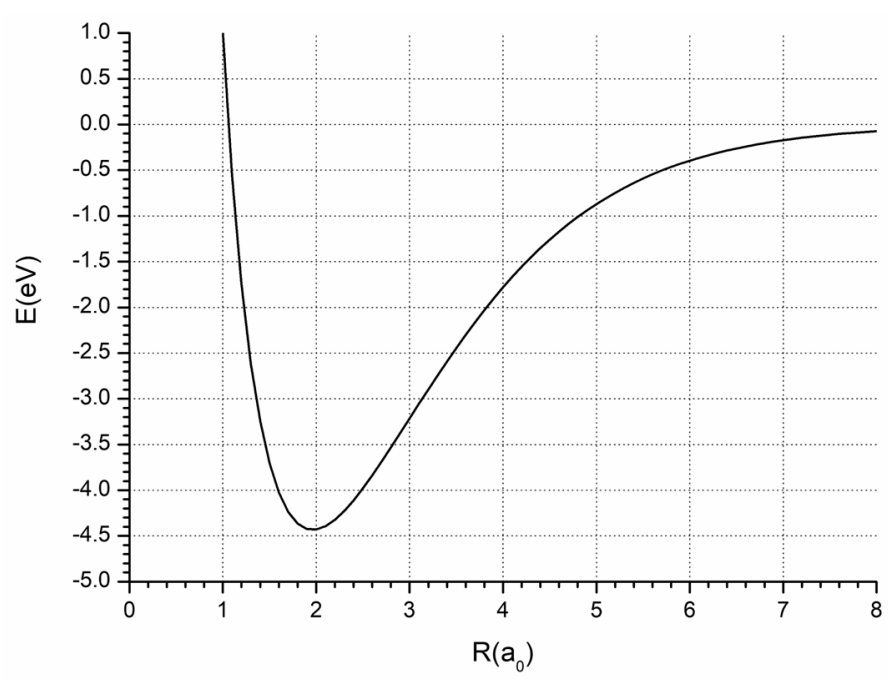

Figure 2. The total energy of the $\mathrm{H}_{2}^{+}$ion $E(R)=T(R)+e \cdot \phi_{H}(R)$ relative to the energy of a free $\mathrm{H}$ atom and a free proton. The distance $R$ between the two protons is in $a_{0}$ units. 


\section{The Central Nucleon-Nucleon Potential Due to Two Pion Exchange}

The effective degrees of freedom in the nuclear interaction at low energy, in particular in the nuclear bound state, are the nucleons and the pions [4] [5] [6] [7] [12] [13] [14] [15]. The intermediate range attraction is due to two pion exchange.

The nuclear interaction which takes place between a neutron and a proton by pion exchange is described by Feynman with similar arguments he used for the covalent bound of $\mathrm{H}_{2}^{+}$ion. The energy of the $\mathrm{H}$ atom is less than that of the proton by $W_{H}$ (calculating nonrelativistically, and omitting the rest energy $m_{e} c^{2}$ of electron), so the electron which penetrates the barrier in the space between the two protons of the molecule has a "negative" kinetic energy. Since in the nuclear process the proton and the neutron have almost equal masses, an exchanged pion will have zero total energy [3]. For a pion of mass $m_{\pi}$ and momentum $p$ the total energy $E$ is:

$$
E^{2}=p^{2} c^{2}+m_{\pi}^{2} c^{4} .
$$

For zero total energy $p$ is again an imaginary number [3]:

$$
p=i m_{\pi} c .
$$

Taking into account the Formula (4) and the Formula (14) the probability amplitude for the pion to jump from one nucleon to another is [3]:

$$
A_{\pi} \sim \mathrm{e}^{-\left(m_{\pi} c / \hbar\right) R}=\mathrm{e}^{-R / \lambda_{\pi}} .
$$

where $\lambda_{\pi}$ is the Compton wavelength of pion. The exponential function, which is typical for a Yukawa potential or exponential potential, limits drastically the probability amplitude for a pion to penetrate the barrier in the space between the two nucleons for large $R$ [3].

Heisenberg was the first who presented the attractive force between proton and neutron in analogy to that in the hydrogen molecular ion $\mathrm{H}_{2}^{+}$. The exchange of electron between the two protons is a real process, the electron is put in common by the two protons. Due to this delocalization the kinetic energy of the electron decreases in the bound system, as shown in Section 2.

We also treat the exchange of pions between the two nucleons like a real process. In consequence, if a pion leaves from nucleon 1 to tunnel to nucleon 2, a pion from nucleon 2 must tunnel simultaneously to nucleon 1, to replace it. A nucleon can emit a real pion only receiving simultaneously an amount of energy equal to the pion mass or directly another pion.

The probability amplitude of simultaneous exchange of the two pions between the nucleons is the product of two probability amplitude of one pion exchange (relation 15):

$$
A_{2 \pi} \sim \mathrm{e}^{-R / \lambda_{\pi}} \cdot \mathrm{e}^{-R / \lambda_{\pi}}=\mathrm{e}^{-2 R / \lambda_{\pi}} .
$$

As it is well known the current masses of quarks (antiquarks) $\mathrm{u}(\overline{\mathrm{u}})$ and $\mathrm{d}(\mathrm{d})$ are very small; the nucleon and pion masses are mainly of dynamical origin 
("kinetic" energy). More than $95 \%$ of the mass of the Universe is "kinetic energy".

The long range structure of the nucleon is given roughly by the pion [12] [13] [14] [15], which also gives the range of nuclear forces. In particular, the Compton wavelength of the pion $\lambda_{\pi}=\hbar / m_{\pi} c$, which has a value of $1.41 \mathrm{fm}$ for the charged pion, gives the range $r_{N}$ of the nucleon extension. The pion in nucleon can be represented by a degree of freedom of current mass $\cong 0$, localized into a region of radius $r_{N}=\lambda_{\pi}$. This localization into the nucleon associates an energy (dynamical mass) $E \cong p c=\hbar c / \lambda_{\pi}$, given by the uncertainty relation, which is just the mass of the pion [9] [10].

When two nucleons approach each other to form a bound state, in particular the deuteron, they put in common some pion degrees of freedom, this means just the two pion exchange process. This is equivalent with a slight delocalization of each pion degree of freedom from a region of linear dimension $r_{N}$ to a region of linear dimension $r_{N}+\Delta(R)$, where $\Delta(R)$ is direct proportional to the distance $R$ between the two bound nucleons and is strongly dependent on the probability amplitude of the two pions to tunnel simultaneously the potential barrier between the two nucleons (Formula (16)). The dynamical mass of each pion degree of freedom gets:

$$
E_{\Delta}=\frac{\hbar c}{\lambda_{\pi}+\Delta(R)} .
$$

and is lower than the initial one (that in the free nucleon: $E=\hbar c / \lambda_{\pi}$ ). The total decrease of the dynamical masses of the two exchanged pions:

$$
\Delta E=2\left(\frac{\hbar c}{\lambda_{\pi}+\Delta(R)}-\frac{\hbar c}{\lambda_{\pi}}\right) .
$$

is just the main contribution to the central nucleon-nucleon potential $V_{2 \pi}(R)$ due to two pion exchange.

As in the case of covalent bonding (see Formula (6)), $\Delta(R)$ is the product between the inter-nucleon distance $R$ and the probability amplitude of the two pions to simultaneously tunnel the potential barrier between the two nucleons (Formula (16)):

$$
\Delta(R)=R \mathrm{e}^{-2 R / \lambda_{\pi}} .
$$

From (18) and (19) it results the following expression of the central nucleon-nucleon potential due to two pion exchange:

$$
V_{2 \pi}(R)=2\left(\frac{\hbar c}{\lambda_{\pi}+R \mathrm{e}^{-2 R / \lambda_{\pi}}}-\frac{\hbar c}{\lambda_{\pi}}\right)=-2 \frac{\hbar c}{\lambda_{\pi}} \cdot \frac{R \mathrm{e}^{-2 R / \lambda_{\pi}}}{\lambda_{\pi}+R \mathrm{e}^{-2 R / \lambda_{\pi}}} .
$$

One notes that $\hbar c / \lambda_{\pi}=m_{\pi} c^{2}$.

This nucleon-nucleon potential $V_{2 \pi}(R)$ due to two pion exchange is shown in Figure 3, for $\lambda_{\pi}=1.41 \mathrm{fm}$ (Compton wavelength of charged pion). The minimum value of the potential is $-44 \mathrm{MeV}$, at $\mathrm{R}=0.7 \mathrm{fm}$, which is comparable to the minimum value $(-50 \mathrm{MeV})$ of the $\mathrm{CD}$ Bonn potential [2]. The fall of the 


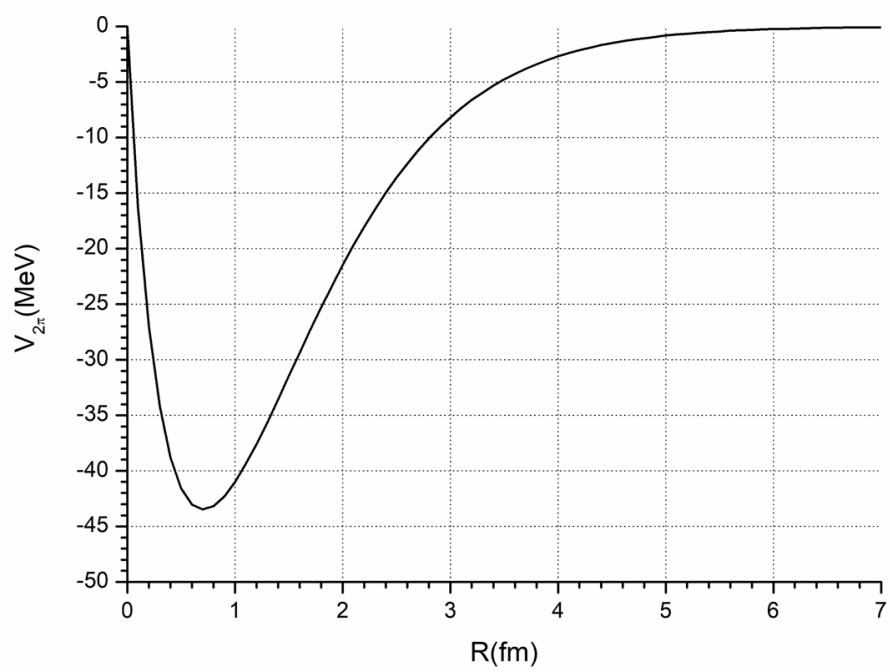

Figure 3. The nucleon-nucleon potential $V_{2 \pi}(R)$ due to two pion exchange as a function of inter-nucleon distance $R$ for $\lambda_{\pi}=1.41 \mathrm{fm}$.

potential for small $R(R<0.6 \mathrm{fm})$ is compatible with the beginning of the hard core repulsion region [4] [5] [6] [7] [13] [16] [17] which gets dominant at short range. The fall of the potential for high values of $R$ is slower than in the case of CD Bonn potential. The width at half maximum is less than $2 \mathrm{fm}$.

Formula (20) of the central nucleon-nucleon potential due to two pion exchange is identical with that obtained in [10]. A hard core repulsion given by an infinite potential for $R \leq r_{0}$, where $r_{0}$ is the hard core radius, was added to the potential $V_{2 \pi}(R)$. The Schrodinger equation for the two nucleons interacting by this two pion exchange potential with hard core repulsion was solved numerically for different values of the hard core radius $r_{0}$ [10]. For a value of the hard core repulsion radius equal to $0.5 \mathrm{fm}$, a typical value for nucleon-nucleon interaction [4] [5] [6] [7] [16] [17], the calculated binding energy is equal to the deuteron binding energy [10]. For pion mass values higher than the real pion mass, with pion mass increase the binding energy decreases, and gets zero at a value of about $190 \mathrm{MeV}$ [10], a result comparable to that obtained in [6]. This underlines the central role of the pions as main player in the production of nuclear attraction.

\section{Discussion and Conclusions}

Feynman made a simple and unitary analysis of the mechanism of both covalent bonding and nucleon-nucleon interaction by particle exchange. In particular, he used the probability amplitude of a particle to get from one place to another a distance $R$ away to describe the behavior of the exchanged particle, in fact its slight delocalization, and assumed that the interaction energy must be proportional to this amplitude.

Starting from this analysis, a unitary quantitative approach to the attraction mechanism in molecular covalent bonding and in central nucleon-nucleon inte- 
raction was made. In our quantitative estimations, only the main ingredients of the interaction mechanism were considered to highlight the main attraction mechanism. Some degrees of freedom, electrons or pions, are put in common between the two protons of the molecule and between the two nucleons respectively. This delocalization, which is strongly limited by the probability amplitude of tunneling of the exchanged particles, determines a decrease in the dynamical mass (kinetic energy) of the system compared to the unbound state.

The decrease in the kinetic energy (dynamical mass from a relativistic point of view) of the exchanged electron in the $\mathrm{H}_{2}^{+}$molecular ion as function of the distance between the protons calculated in the present work is similar to that derived by detailed quantum mechanics calculus. The relation (9), which represents the attractive part of the potential for the molecular ion $\mathrm{H}_{2}^{+}$, can be written as follows:

$$
T(R)=-\frac{\hbar^{2}}{2 m_{e} a_{0}^{2}}\left(x \mathrm{e}^{-x}\right) \frac{2+x \mathrm{e}^{-x}}{\left(1+x \mathrm{e}^{-x}\right)^{2}} \equiv-E_{k i n, H}\left(x \mathrm{e}^{-x}\right) \frac{2+x \mathrm{e}^{-x}}{\left(1+x \mathrm{e}^{-x}\right)^{2}}
$$

where $x=R / a_{0}$ and $E_{k i n, H}$ is given by relation (8). Since the maximum value of $x \mathrm{e}^{-x}$ is 0.368 , for $x=1\left(R=a_{0}\right)$, the main contribution to the variation of $T$ with distance is given by the term in parenthesis $x \mathrm{e}^{-x}$. This dependence is similar to the attractive part of the Rydberg potential used to describe the molecular covalent bond [18].

The decrease in the dynamical mass of the two pions exchanged simultaneously between the two nucleons is the principal mechanism responsible for the nucleon-nucleon attraction. A pion from nucleon 1 tunnels to nucleon 2 and simultaneously a pion from nucleon 2 tunnels to nucleon 1 . The central nucleon-nucleon potential obtained in this approach (Formula (20)) has a minimum value comparable to that of the CD Bonn potential and has a fall towards zero value for small values of $R$ compatible with the beginning of the hard core repulsion region. Relation (20) can be written as follows:

$$
V_{2 \pi}(R)=-2 \frac{\hbar c}{\lambda_{\pi}} \cdot \frac{\frac{R}{\lambda_{\pi}} \mathrm{e}^{-2 R / \lambda_{\pi}}}{1+\frac{R}{\lambda_{\pi}} \mathrm{e}^{-2 R / \lambda_{\pi}}} \equiv-m_{\pi} c^{2}\left(x \mathrm{e}^{-x}\right) \frac{1}{1+\frac{x}{2} \mathrm{e}^{-x}}
$$

where $x=2 R / \pi_{\pi}$. Since the maximum value of $x \mathrm{e}^{-x} / 2$ is 0.18 , for $x=1$ ( $R=\lambda_{\pi} / 2$ ), the same dependence $x \mathrm{e}^{-x}$ (the term in parenthesis) is dominant in the nucleon-nucleon attraction mechanism, like in the case of covalent bond. It looks like a universal attraction mechanism for the two interactions.

The pion is part of the nucleon structure. In particular, the Lattice QCD calculations have shown that the nucleon mass displays a linear dependence on the pion mass, known as the "ruler approximation" [19]:

$$
m_{N}(\mathrm{MeV})=800+m_{\pi}
$$

This means that the decrease in the mass of the exchanged pions in the nucleon-nucleon interaction, actually means the decrease in the mass of the 
nucleons. But this nucleon mass decrease is a manifestation of the partial chiral symmetry restoration in nuclear matter [20]. There is a direct relationship between the slight delocalization of the pion degrees of freedom and the mass decrease of the nucleons which exchange those pions.

Let's analyze at the quark level. Because the pions are made up of quarks and antiquarks, by pion exchange some quark degrees of freedom are implicitly exchanged between the two nucleons and, consequently, are slightly delocalized. This means that the confinement region of some quarks slightly increases and accordingly their dynamical mass decreases. It could be said that there is a slight deconfinement of some quark degrees of freedom and, therefore, the mass of nucleons decreases.

\section{Acknowledgements}

The author expresses his sincere thanks to E. Dudas, S. Nordholm and D. I. Palade for helpful discussions and suggestions.

\section{Conflicts of Interest}

The author declares no conflicts of interest regarding the publication of this paper.

\section{References}

[1] Bacskay, G.B. and Nordholm, S. (2013) The Journal of Physical Chemistry A, 117, 33. https://doi.org/10.1021/jp403284g

[2] Rioux, F. (1997) Journal of Chemical Education, 2, 1.

[3] Feynman, R., Leighton, R. and Sands, M. (1964) Feynman Lectures on Physics. Vol. 3, Quantum Mechanics. Addison-Wesley, Boston, Chapters 3, 7 and 10.

[4] Machleidt, R. (2007) Lectures 1-4, "Nuclear Forces".

[5] Ericson, T. and Weise, W. (1988) Pions and Nuclei. Clarendon Press, Oxford.

[6] Epelbaum, E., Hammer, H.W. and Meissner, U.-G. (2008) Modern Theory of Nuclear Forces.

[7] Machleidt, R. (2013) Origin and Properties of Strong Inter-Nucleon Interactions.

[8] Mandache, N.B. (2009) Anomalous Delta-Type Electric and Magnetic Two-Nucleon Interactions. https://arxiv.org/abs/0904.1080

[9] Mandache, N.B. (2012) Romanian Reports in Physics, 64, 1307.

[10] Mandache, N.B. and Palade, D.I. (2018) Journal of Modern Physics, 9, 1459. https://doi.org/10.4236/jmp.2018.98090

[11] Jackson, J.D. (1998) Classical Electrodynamics. John Wiley \& Sons, Inc., Hoboken.

[12] Thomas, A.W., Theberge, S. and Miller, G.A. (1981) Physical Review D, 24, 216. https://doi.org/10.1103/PhysRevD.24.216

[13] Brown, G.E. and Rho, M. (1983) Physics Today, 36, 24. https://doi.org/10.1063/1.2915491

[14] Thomas, A.W. and Weise, W. (2001) The Structure of the Nucleon. Wiley-VCH, Hoboken. https://doi.org/10.1002/352760314X

[15] Vanderhaeghen, M. and Walcher, T. (2010) Long Range Structure of the Nucleon. 
[16] Ishii, N., Aoki, S. and Hatsuda, T. (2007) Physical Review Letters, 99, Article ID: 022001. https://doi.org/10.1103/PhysRevLett.99.022001

[17] Aoki, S., Doi, T., Hatsuda, T., Ikeda, Y., Inoue, T., Ishii, N., Murano, K., Nemura, H. and Sasaki, K. (2012) Progress of Theoretical and Experimental Physics, 2012, 01 A105. https://doi.org/10.1093/ptep/pts010

[18] Varshni, Y.P. (1957) Reviews of Modern Physics, 29, 664. https://doi.org/10.1103/RevModPhys.29.664

[19] Walker-Loud, A. (2013) Baryons in/and Lattice QCD.

[20] Birse, M. and McGovern, J. (1995) Physics World, 10, 35. https://doi.org/10.1088/2058-7058/8/10/29 